\title{
Synthesis of Superparamagnetic Iron Oxide Nanoparticles Modified with MPEG-PEI via Photochemistry as New MRI Contrast Agent
}

\author{
Yancong Zhang, Lianying Zhang, Xinfeng Song, Xiangling Gu, \\ Hanwen Sun, Chunhua Fu, and Fanzong Meng
}

Shandong Provincial Engineering Laboratory of Novel Pharmaceutical Excipients, Sustained and Controlled Release Preparations, Dezhou University, Dezhou 253023, China

Correspondence should be addressed to Hanwen Sun; hanwen916@163.com

Received 24 September 2014; Revised 11 January 2015; Accepted 25 January 2015

Academic Editor: Paulo Cesar Morais

Copyright (C) 2015 Yancong Zhang et al. This is an open access article distributed under the Creative Commons Attribution License, which permits unrestricted use, distribution, and reproduction in any medium, provided the original work is properly cited.

\begin{abstract}
Novel method for synthesis of superparamagnetic iron oxide nanoparticles (SPIONs) coated with polyethylenimine (PEI) and modified with poly(ethylene glycol) methyl ether (MPEG), MPEG-PEI-SPIONs, was developed. PEI-SPIONs were successfully prepared in aqueous system via photochemistry, and their surface was modified with poly(ethylene glycol) methyl ether (MPEG). The so-obtained MPEG-PEI-SPIONs had a uniform hydrodynamic particle size of $34 \mathrm{~nm}$. The successful coating of MPEG-PEI on the SPIONs was ascertained from FT-IR analysis, and the PEI and MPEG fractions in MPEG-PEI-SPIONs were calculated to account for $31 \%$ and $12 \%$, respectively. Magnetic measurement revealed that the saturated magnetization of MPEG-PEI-SPIONs reached $46 \mathrm{emu} / \mathrm{g}$ and the nanoparticles showed the characteristic of being superparamagnetic. The stability experiment revealed that the MPEG-PEI modification improved the nanoparticles stability greatly. T2 relaxation measurements showed that MPEGPEI-SPIONs show similar $R 2$ value to the PEI-SPIONs. The T2-weighted magnetic resonance imaging (MRI) of MPEG-PEISPIONs showed that the magnetic resonance signal was enhanced significantly with increasing nanoparticle concentration in water. These results indicated that the MPEG-PEI-SPIONs had great potential for application in MRI.
\end{abstract}

\section{Introduction}

Magnetic resonance imaging (MRI) is a noninvasive technique routinely used in clinics for diagnostic imaging. It has become very important diagnostic modality in hospitals [1]. The quality of the MRI images depends on three NMR main parameters (the proton spin density, the nuclear spin-lattice relaxation time $T 1$, and the spin-spin relaxation time $T 2$ ), and the contrast agents (CAs), based on the different distribution of nuclear-spin density along the body, are able to improve the image contrast by increasing (locally) the nuclear relaxation rates [2].

Superparamagnetic nanoparticles are represented as $T 2$ (transversal relaxation time) contrast agents as opposed to $T 1$ (longitudinal relaxation time) contrast agents such as paramagnetic gadolinium(III) chelates $[3,4]$. The superparamagnetic iron oxide nanoparticles (SPIONs) are the most commonly used superparamagnetic contrast agents. During the preparation, storage, and application of MR contrast agent, the stability and biocompatibility of SPIONs are important [5]. However, due to the high ratio of surface to volume and magnetization, SPIONs are prone to aggregate in water or tissue fluid which limits the application [6]. To reduce aggregation and enhance the biocompatibility, the coating of polymer onto SPIONs surface is indispensable [7, 8]. When coated with polymer, SPIONs have a reduced aggregation, improved biocompatibility, and longer half-life in circulation and are successfully used for MRI contrast agent and targeted gene and drug delivery [9-11].

Polyethylenimine (PEI) is a water soluble cationic polymer which contains amino and imino groups in each polymer chain. When the polymer PEI is dispersed in aqueous solution, each polymer chain is positively charged by amino and imino groups [12]. The dispersibility of the composite 
nanoparticles is expected to be enhanced by the electrostatic repulsive force and steric hindrance of the PEI polymers [13]. Corti et al. [14] synthesized polyethylenimine-coated superparamagnetic nanoparticles and demonstrated their ability to be used as MRI contrast agents. However, the toxicity of PEI has limited its use, particularly for in vivo application [15].

Poly(ethylene glycol) (PEG) has been employed extensively in pharmaceutical and biomedical fields because of its outstanding physicochemical and biological properties including hydrophilic property, solubility, nontoxicity, ease of chemical modification, and absence of antigenicity and immunogenicity [16]. Therefore, PEG is widely used as a pharmacological polymer with high hydrophilicity, biocompatibility, and biodegradability. It is ideal for prevention of bacterial surface growth, decrease of plasma protein binding and erythrocyte aggregation, and prevention of recognition by the immune system.

In recent years, PEG modified PEI has been synthesized for gene delivery $[17,18]$. However, to the best of our knowledge, the PEG modified PEI coated superparamagnetic iron oxide nanoparticles have not been synthesized for MRI contrast agents. In this report, we describe the synthesis of PEI coated SPIONs via photochemistry and surface modified with poly(ethylene glycol) methyl ether (MPEG). The physical properties, stability, and MRI of the MPEG-PEI-SPIONs are investigated and the results showed that these novel nanoparticles may be a good candidate for bioapplications utilizing MRI.

\section{Materials and Methods}

2.1. Materials. Polyethylenimine (PEI, average Mn 400) and $1,1^{\prime}$-carbonyldiimidazole (CDI), analytical grade, were purchased from Tokyo Chemical Industry Co. Ltd., Japan. Poly(ethylene glycol) methyl ether (MPEG, average Mn 550) was purchased from Sigma-Aldrich, USA. Ferrous chloride tetrahydrate $\left(\mathrm{FeCl}_{2} \cdot 4 \mathrm{H}_{2} \mathrm{O}\right)$, ferric chloride hexahydrate $\left(\mathrm{FeCl}_{3} \cdot 6 \mathrm{H}_{2} \mathrm{O}\right.$ ), and $\mathrm{NH}_{3}$ aqueous solution (25 wt\%), analytical grade, were purchased from Shanghai Chemical Reagents Company (China) and used without further treatment. Nitrogen (99.99\%) was available from Dezhou LongLi Company, China. All the other chemicals were of analytical grade.

Stirrer (IKA Company, Germany) was used to synthesize the $\mathrm{Fe}_{3} \mathrm{O}_{4}$ nanoparticles (SPIONs). A photochemical reaction device equipped with two $8 \mathrm{~W}$ low-pressure mercury lamps, which had been described in detail in our previous papers $[19,20]$, was used as the UV source to synthesize PEI-SPIONs.

2.2. Synthesis of $\mathrm{Fe}_{3} \mathrm{O}_{4}$ Nanoparticles: SPIONs. Superparamagnetic magnetic nanoparticles were prepared via improved chemical coprecipitation method [21, 22]. Briefly, $3.91 \mathrm{~g} \mathrm{FeCl}_{3} \cdot 6 \mathrm{H}_{2} \mathrm{O}$ and $1.43 \mathrm{~g} \mathrm{FeCl}_{2} \cdot 4 \mathrm{H}_{2} \mathrm{O}$ were dissolved in $150 \mathrm{~mL}$ deionized water in a $500 \mathrm{~mL}$ beaker under nitrogen gas and vigorously stirred at $85^{\circ} \mathrm{C}$ until dissolved, after which $7 \mathrm{~mL}$ of $\mathrm{NH}_{3}$ aqueous solution was injected into the solution rapidly. After 1 hour of stirring at $85^{\circ} \mathrm{C}$, the SPIONs precipitated and were isolated from the solution by a permanent magnet (4,000 Gaus). The SPIONs were washed five times with deionized water and separated by magnetic decantation. Finally, the SPIONs were dispersed in $100 \mathrm{~mL}$ deionized water and stored at $4^{\circ} \mathrm{C}$.

2.3. Preparation of PEI-SPIONs. The PEI coated SPIONs were synthesized in a $150 \mathrm{~mL}$ interlayer quartz flask equipped with a stirrer and $\mathrm{N}_{2}$ inlet cooling with cycled water. $100 \mu \mathrm{L}$ of PEI and $10 \mathrm{mg}$ SPIONs were mechanically mixed in $60 \mathrm{~mL}$ water for $30 \mathrm{~min}$, after which $1 \mathrm{~mL}$ of $\mathrm{H}_{2} \mathrm{O}_{2}$ was added, and then the mixture was irradiated by two $8 \mathrm{~W}$ lamps for $20 \mathrm{~min}$ and $\mathrm{N}_{2}$ was bubbled throughout the preparation process. The PEISPIONs were collected with a permanent magnet and washed with deionized water for several times and at last redispersed in deionized water.

2.4. Modification of PEI-SPIONs with MPEG. To a solution of $400 \mu \mathrm{L}$ MPEG in $15 \mathrm{~mL}$ dry acetonitrile under $\mathrm{N}_{2}$ was added $1.5 \mathrm{~g}$ of CDI, and the solution was stirred at $40^{\circ} \mathrm{C}$ for 4 hours. $30 \mathrm{mg}$ of PEI-SPIONs (dissolved in $30 \mathrm{~mL}$ water) was added into the solution and the mixture was stirred for another 2 hours. At last, the MPEG-PEI-SPIONs were collected with a magnet and washed with deionized water for 3 times.

2.5. Characterization. FT-IR spectra of MPEG-PEI-SPIONs were analyzed with a Nicolet Avatar 370 Fourier transform infrared spectrometer (FT-IR) in a wave number range of $4000-500 \mathrm{~cm}^{-1}$. Structure of the magnetic nanoparticles was observed with transmission electron microscopy (TEM, Philips, CM120). The average size in aqueous system was detected by photon correlation spectroscopy (PCS, Zetasizer Nano ZS, Malvern Instruments Ltd.). Thermogravimetric analysis (TG) was done by a thermal analyzer (DTG-60, Shimadzu) by heating the sample from room temperature to $900^{\circ} \mathrm{C}$ under $\mathrm{N}_{2}$ atmosphere at a heating rate of $10^{\circ} \mathrm{C} \mathrm{min}{ }^{-1}$. Saturated magnetization of the nanoparticles was determined on vibrating sample magnetometer (VSM, Model 155, Princeton Applied Research) at room temperature. The samples for FT-IR, TEM, and TG were vacuum-dried at room temperature. The sample for PCS was diluted to $10^{-5} \mathrm{molL}^{-1}$.

MRI experiments were performed at $25^{\circ} \mathrm{C}$ in a clinical magnetic resonance (MR) scanner (NIUMAG, 0.5T, Shanghai, China). To demonstrate the $T 2$ effects in an aqueous solution, MPEG-PEI-SPIONs were suspended in tubes of water $(20 \mathrm{~mL})$ with different SPION concentrations. The tubes were placed into the MR scanner and a number of MR sequences were run, spin-echo for $R 2$ (32 echoes, TR: $3000 \mathrm{~ms}$, TE: 0 4000 ms) determination.

\section{Results and Discussions}

3.1. Synthesis of MPEG-PEI-SPIONs. According to our previous studies, the SPIONs could adsorb PEI molecules on their surface due to the high ratio of surface to volume when SPIONs were dispersed in aqueous solution containing PEI $[19,20]$. The $\mathrm{H}_{2} \mathrm{O}_{2}$ could be decomposed into $\bullet \mathrm{OH}$, which could initiate the PEI to generate free radicals when the solution was exposed to UV irradiation [23]. PEI chains were grafted from the SPIONs via recombination of the PEI free radicals, and then the surface of $\mathrm{Fe}_{3} \mathrm{O}_{4}$ nanoparticles 
was coated by a cross-linked PEI shell via further crosslinking. The PEI-SPIONs were surface-modified with MPEG by covalently binding CDI-activated MPEG. The preparation route of MPEG-PEI-SPIONs was illustrated in Figure 1.

Figure 2 shows the FT-IR spectra of (a) MPEG-PEISPIONs and (b) naked SPIONs. The $1644 \mathrm{~cm}^{-1}$ bond was correlated to the scissoring vibration of $\mathrm{N}-\mathrm{H}$ bond of $-\mathrm{NH}_{2}$ from PEI, and the $1550 \mathrm{~cm}^{-1}$ bond was correlated to the carbodiimide. The bands of $1360 \mathrm{~cm}^{-1}$ and $1102 \mathrm{~cm}^{-1}$ were assigned to the $\mathrm{C}-\mathrm{H}$ bond of $-\mathrm{CH}_{3}$ and $\mathrm{C}-\mathrm{O}-\mathrm{C}$ bound of $-\left(\mathrm{OCH}_{2} \mathrm{CH}_{2}\right)_{n} \mathrm{CH}_{3}$, respectively. Furthermore, the peak of $593 \mathrm{~cm}^{-1}$ in curves (a) and (b) was the characteristic absorption of $\mathrm{Fe}-\mathrm{O}$ bound. All what is above confirmed that the MPEG-PEI-SPIONs were successfully prepared under UV irradiation.

Thermogravimetric analysis (TG) was carried out for the SPIONs, PEI-SPIONs, and MPEG-PEI-SPIONs. The SPIONs, PEI-SPIONs, and MPEG-PEI-SPIONs gave their distinctive TG curves, which provide indications of the amount of PEI and MPEG on the SPIONs. In Figure 3(a), the weight loss from 120 to $700^{\circ} \mathrm{C}$ was about $5 \%$ for SPIONs. In Figure 3(b), the weight loss from 120 to $700^{\circ} \mathrm{C}$ was about $36 \%$. Considering the weight loss of SPIONs, the weight of the PEI fraction in PEI-SPIONs was calculated to account for $31 \%$ of the total weight. Figure 3(c) shows the TG curve of MPEGPEI-SPIONs. The weight loss of the MPEG-PEI-SPIONs from 120 to $700^{\circ} \mathrm{C}$ was about $48 \%$. Considering the weight loss of SPIONs and PEI-SPIONs, the weight of the MPEG fraction in MPEG-PEI-SPIONs was calculated to account for $12 \%$ of the total weight.

3.2. Structure, Size, and Magnetic Properties of MPEGPEI-SPIONs. Morphology and structure of the MPEG-PEISPIONs were studied by TEM. As shown in Figure 4, the MPEG-PEI-SPIONs were of nearly spherical shape. The average size in aqueous system measured by PCS was $34 \mathrm{~nm}$ with a polydispersion index of 0.276 (Figure 5). As the diameter is smaller than $50 \mathrm{~nm}$, the MPEG-PEI-SPIONs can avoid the phagocytosis of reticuloendothelial system (RES) and are designed to have a much longer blood half-life and could rapidly circulate almost the whole body. Thus, the MPEGPEI-SPIONs can penetrate deep tissue spaces (such as the interior of atherosclerotic plaque and the myocardium), and the hydrophilic surface may prolong the circulation in blood $[24,25]$.

The magnetic properties of the naked SPIONs, PEISPIONs, and MPEG-PEI-SPIONs were studied by vibration sample magnetometer (VSM). The saturation magnetization (Ms) value of naked SPIONs was $68 \mathrm{emu} / \mathrm{g}$. After PEI coating, the Ms decreased to $55 \mathrm{emu} / \mathrm{g}$ due to the decrease in SPIONs content of the nanoparticles. After MPEG modification, the Ms decreased to $46 \mathrm{emu} / \mathrm{g}$. This level of $\mathrm{Ms}$ is deemed sufficient for bioapplications where Ms of $7-22 \mathrm{emu} / \mathrm{g}$ is usually adopted $[26,27]$. No hysteresis was observed in Figure 6, indicating the nanoparticles are superparamagnetic at room temperature. Thus, the MPEG-PEI-SPIONs possess superparamagnetism which are suitable for MR contrast enhancement by alterations of proton relaxation in the tissue microenvironment [28].

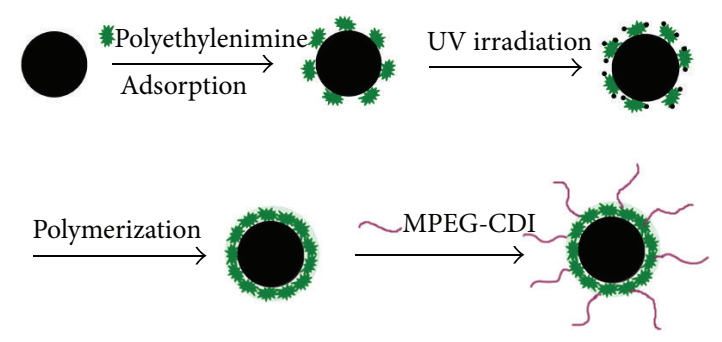

FIGURE 1: Schematic illustration of the preparation route of MPEIPEI-SPIONs.

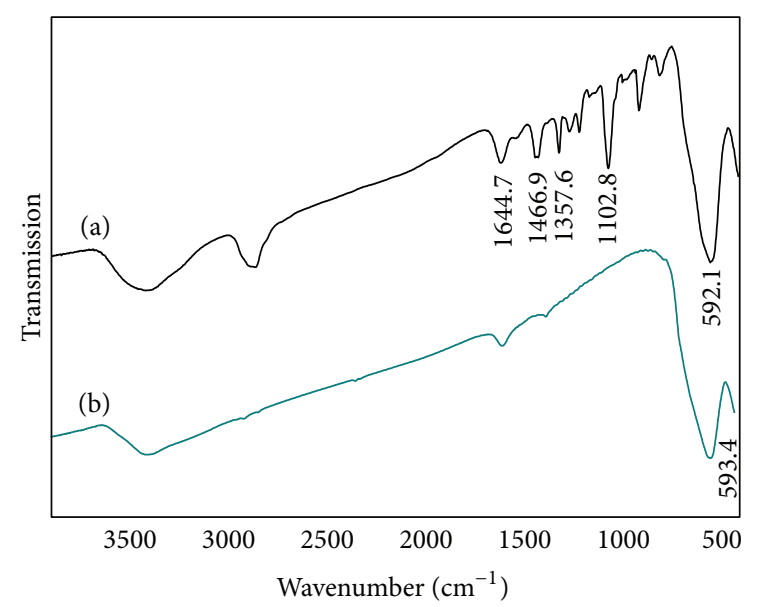

FIGURE 2: FT-IR spectra of the (a) MPEG-PEI-SPIONs and (b) naked SPIONs.

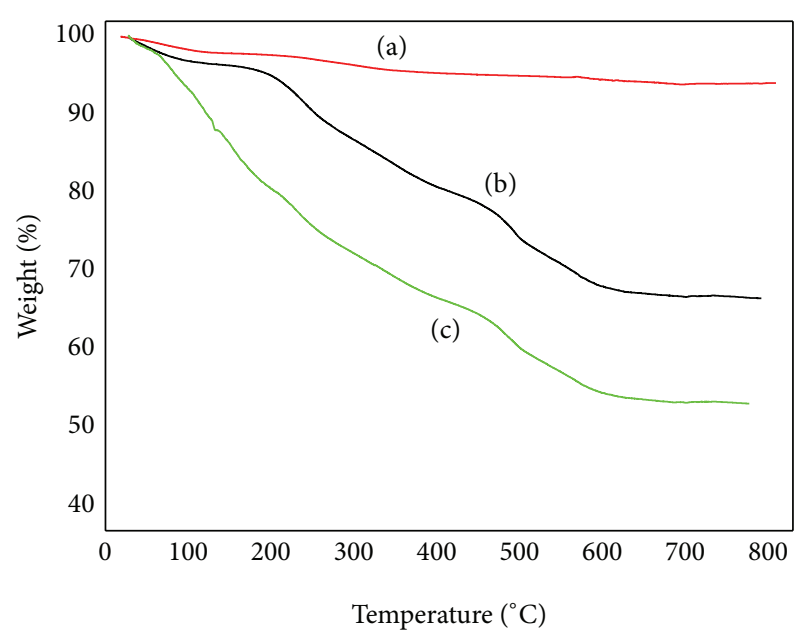

FIgure 3: The TG curve of the (a) naked SPIONs, (b) PEI-SPIONs, and (c) MPEG-PEI-SPIONs.

3.3. Stability. High stability is an important parameter, which determined whether MPEG-PEI-SPIONs can be applied in tissue imaging. The magnetic nanoparticles dissolved in PBS ( $\mathrm{pH} 7.0)$ were stored at $4^{\circ} \mathrm{C}$ and the supernatant was sampled at different intervals to investigate the stability using ultraviolet-visible spectrophotometry (UV-1800, Shimadzu). The optical density (OD) was detected at $660 \mathrm{~nm}$ [29], at 


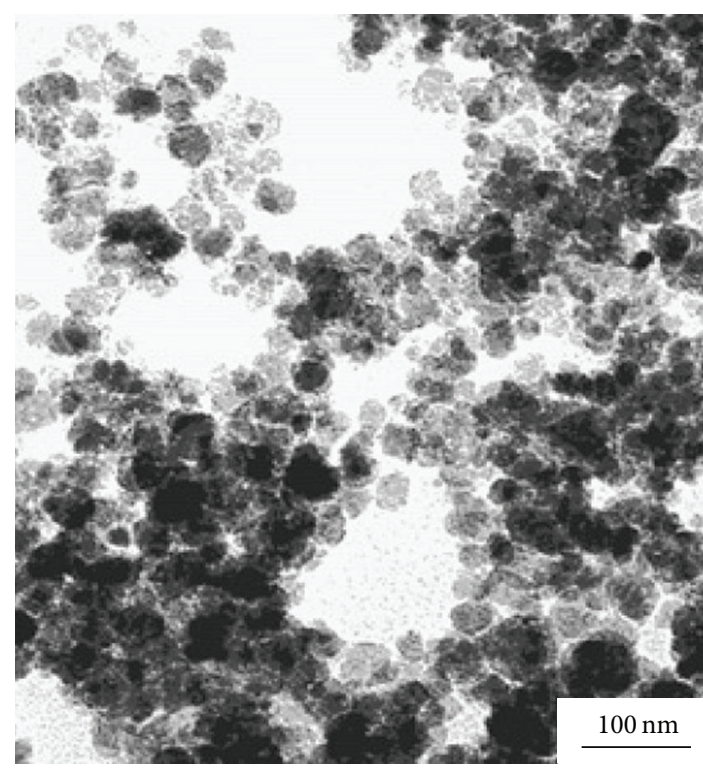

FIgURE 4: The TEM image of MPEG-PEI-SPIONs.

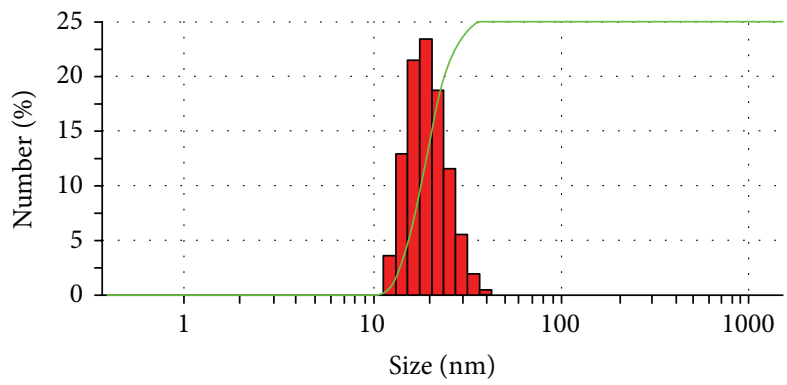

FIgure 5: Particle size of the MPEG-PEI-SPIONs measured by PCS.

which the OD value was linearly related to the concentrations of magnetic nanoparticles. Stability was calculated according to

$$
\text { Stability }(\%)=\frac{\mathrm{OD}_{s}}{\mathrm{OD}_{f}} \times 100 \%,
$$

where $\mathrm{OD}_{s}$ was $\mathrm{OD}$ value of nanoparticles stored for certain time and $\mathrm{OD}_{f}$ was $\mathrm{OD}$ value of the freshly prepared sample. The result was shown in Figure 7. The PEI coating could improve the stability of the SPIONs in the observed 178 hours. After MPEG modification, the MPEG-PEI-SPIONs have a higher stability in PBS ( $\mathrm{pH} 7.0)$, as they could retain $88.7 \%$ in aqueous suspension over a week. The high stability is due to the electrostatic repulsive force and steric hindrance of the PEI polymers against aggregation, and the high hydrophilicity of MPEG may also contribute to the stability.

3.4. MR Imaging. To investigate the $T 2$ relaxation properties of SPIONs, PEI-SPIONs, and MPEG-PEI-SPIONs, T2 relaxation measurements had been made using $0.5 \mathrm{~T}$ MR imager. Figure 8 shows the $T 2$ relaxation curves for the three samples. The naked SPIONs showed faster $T 2$ decay curve compared

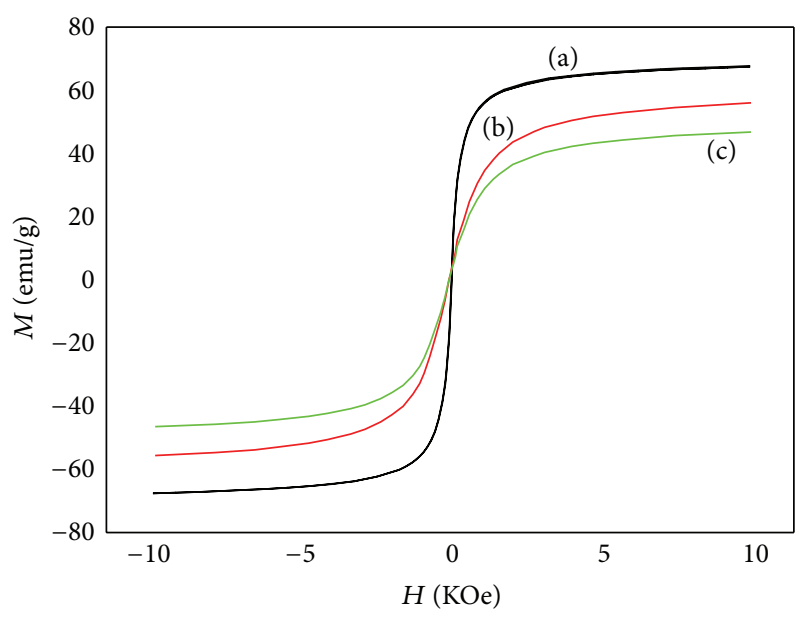

FIgure 6: Magnetization curve of the (a) naked SPIONs, (b) PEISPIONs, and (c) MPEG-PEI-SPIONs.

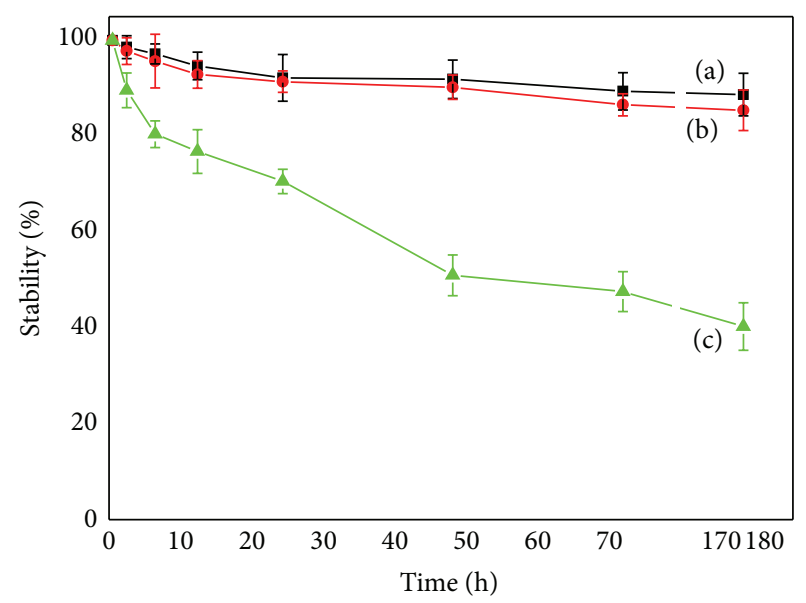

FIGURE 7: Stability of the (a) MPEI-PEI-SPIONs, (b) PEI-SPIONs, and (c) naked SPIONs in vitro (retention ratio versus stored time).

to PEI-SPIONs and MPEG-PEI-SPIONs. The MPEG-PEISPIONs had a similar T2 decay curve. Thus, the MPEGPEI-SPIONs nanoparticles show similar $R 2(=1 / T 2)$ value with the PEI-SPIONs. This result indicates that the MPEG modification does not decrease the $T 2$ relaxation properties compared with PEI-SPIONs.

Finally, we performed MRI experiments on a phantom composed of four sample tubes containing MPEGPEI-SPIONs in water at different concentrations (spin-echo technique with $\mathrm{TR}=3000 \mathrm{~ms}$ and $\mathrm{TE}=100 \mathrm{~ms}$ ). It can be seen from Figure 9 that the MR signal intensity (related to the $T 2$ relaxation time in $T 2$-weighted image) for the samples of different concentrations is not identical. With increasing MPEG-PEI-SPIONs concentration in water, the MR signal was enhanced significantly (negative in brightness in T2weighted image). This result indicates that the nanoparticles can generate high magnetic field gradients near the surface of the MPEG-PEI-SPIONs. 


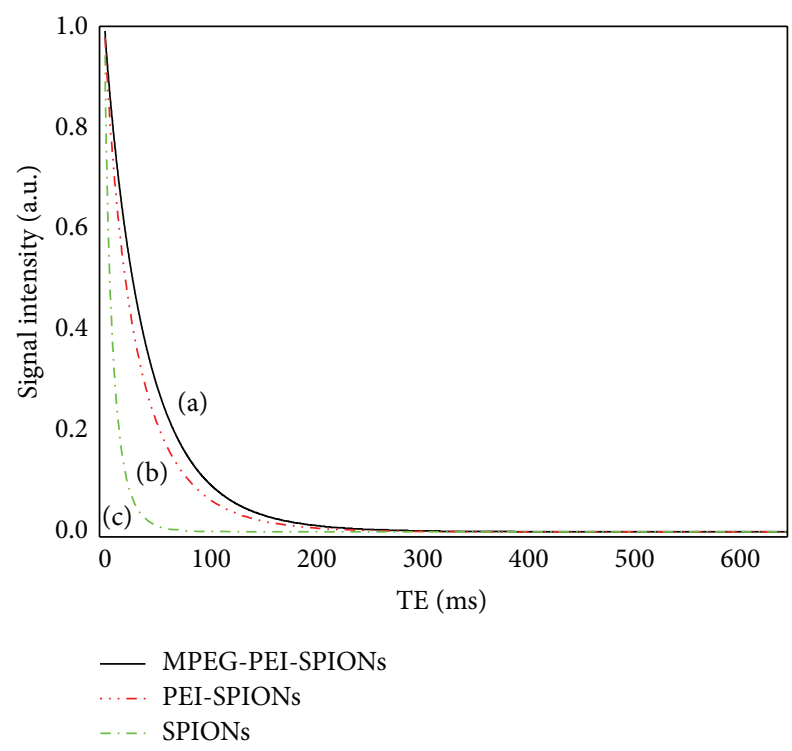

FIGURE 8: The T2 relaxation curves for (a) MPEI-PEI-SPIONs, (b) PEI-SPIONs, and (c) naked SPIONs.

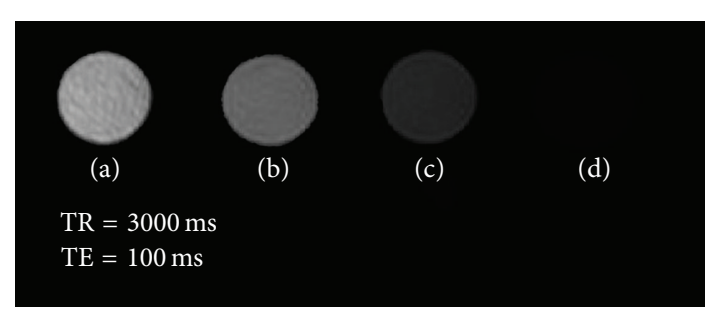

FIGURE 9: T2-weighted MRI images of MPEG-PEI-SPIONs with different concentrations. (a) 0.063 , (b) 0.125 , (c) 0.250 , and (d) $0.500 \mathrm{mg} / \mathrm{mL}$.

\section{Conclusions}

In summary, PEI coated SPIONs were successfully synthesized via photochemistry method, and the MPEG-PEISPIONs were prepared with surface MPEG modification of PEI-SPIONs. The analyses of TEM and PCS indicated that the MPEG-PEI-SPIONs were nearly spherical in shape, and the mean diameter in aqueous solution was $34 \mathrm{~nm}$. These novel nanoparticles are superparamagnetic with $\mathrm{Ms}$ of $46 \mathrm{emu} / \mathrm{g}$ and possess good stability in water. The surface modification by MPEG-PEI polymers could greatly enhance SPIONs' stability. The MPEG-PEI-SPIONs showed similar $R 2$ value with the PEI-SPIONs and could generate high magnetic field gradients near the surface of the MPEG-PEI-SPIONs. High stability, biocompatibility, and higher $R 2$ value promised the MPEG-PEI-SPIONs would be an ideal candidate for potential MRI contrast agents. Further MRI tests on specific models of different pathologies are in progress.

\section{Conflict of Interests}

The authors declare that there is no conflict of interests regarding the publication of this paper.

\section{Acknowledgments}

The authors are grateful to the National Natural Science Foundation of China (no. 51273033), Projects of Medical and Health Technology Development Program in Shandong Province (no. 2011HZ005), Promotive Research Fund for Young and Middle-aged Scientists of Shandong Province (BS2014HZ007), Project of Shandong Province Higher Educational Science and Technology Program (no. J14LC56), Shandong Provincial Development Project of Science and Technology (no. 2014GGX102037), and Shandong Province Young and Middle-Aged Scientists Research Awards Fund (no. BS2011CL001).

\section{References}

[1] M. G. Lansberg, M. Straka, S. Kemp et al., "MRI profile and response to endovascular reperfusion after stroke (DEFUSE 2): a prospective cohort study," The Lancet Neurology, vol. 11, no. 10, pp. 860-867, 2012.

[2] D. E. Sosnovik, M. Nahrendorf, and R. Weissleder, "Molecular magnetic resonance imaging in cardiovascular medicine," Circulation, vol. 115, no. 15, pp. 2076-2086, 2007.

[3] T. K. Jain, J. Richey, M. Strand, D. L. Leslie-Pelecky, C. A. Flask, and V. Labhasetwar, "Magnetic nanoparticles with dual functional properties: drug delivery and magnetic resonance imaging," Biomaterials, vol. 29, no. 29, pp. 4012-4021, 2008.

[4] C. Corot, P. Robert, J.-M. Idée, and M. Port, "Recent advances in iron oxide nanocrystal technology for medical imaging," Advanced Drug Delivery Reviews, vol. 58, no. 14, pp. 1471-1504, 2006.

[5] S. Yoffe, T. Leshuk, P. Everett, and F. Gu, "Superparamagnetic iron oxide nanoparticles (SPIONs): synthesis and surface modification techniques for use with MRI and other biomedical applications," Current Pharmaceutical Design, vol. 19, no. 3, pp. 493-509, 2013.

[6] J. S. Nunes, C. L. de Vasconcelos, F. A. O. Cabral, J. H. de Araújo, M. R. Pereira, and J. L. C. Fonseca, "Synthesis and characterization of poly(ethyl methacrylate-co-methacrylic acid) magnetic particles via miniemulsion polymerization," Polymer, vol. 47, no. 22, pp. 7646-7652, 2006.

[7] R. Y. Hong, J. H. Li, J. M. Qu, L. L. Chen, and H. Z. Li, “Preparation and characterization of magnetite/dextran nanocomposite used as a precursor of magnetic fluid," Chemical Engineering Journal, vol. 150, no. 2-3, pp. 572-580, 2009.

[8] R. Y. Hong, B. Feng, L. L. Chen et al., "Synthesis, characterization and MRI application of dextran-coated $\mathrm{Fe}_{3} \mathrm{O}_{4}$ magnetic nanoparticles," Biochemical Engineering Journal, vol. 42, no. 3, pp. 290-300, 2008.

[9] R. Chen, H. Yu, Z.-Y. Jia, Q.-L. Yao, and G.-J. Teng, "Efficient nano iron particle-labeling and noninvasive MR imaging of mouse bone marrow-derived endothelial progenitor cells.", International Journal of Nanomedicine, vol. 6, pp. 511-519, 2011.

[10] J. S. Guthi, S.-G. Yang, G. Huang et al., "MRI-visible micellar nanomedicine for targeted drug delivery to lung cancer cells," Molecular Pharmaceutics, vol. 7, no. 1, pp. 32-40, 2010.

[11] C. Wang, S. Ravi, G. V. Martinez et al., "Dual-purpose magnetic micelles for MRI and gene delivery," Journal of Controlled Release, vol. 163, no. 1, pp. 82-92, 2012. 
[12] F. Tang, T. Uchikoshi, K. Ozawa, and Y. Sakka, "Effect of polyethylenimine on the dispersion and electrophoretic deposition of nano-sized titania aqueous suspensions," Journal of the European Ceramic Society, vol. 26, no. 9, pp. 1555-1560, 2006.

[13] S. Seino, Y. Matsuoka, T. Kinoshita, T. Nakagawa, and T. A. Yamamoto, "Dispersibility improvement of gold/iron-oxide composite nanoparticles by polyethylenimine modification," Journal of Magnetism and Magnetic Materials, vol. 321, no. 10, pp. 1404-1407, 2009.

[14] M. Corti, A. Lascialfari, M. Marinone et al., "Magnetic and relaxometric properties of polyethylenimine-coated superparamagnetic MRI contrast agents," Journal of Magnetism and Magnetic Materials, vol. 320, no. 14, pp. e316-e319, 2008.

[15] M. Thomas, Q. Ge, J. J. Lu, J. Chen, and A. M. Klibanov, "Cross-linked small polyethylenimines: while still nontoxic, deliver DNA efficiently to mammalian cells in vitro and in vivo," Pharmaceutical Research, vol. 22, no. 3, pp. 373-380, 2005.

[16] P. Kolhe and R. M. Kannan, "Improvement in ductility of chitosan through blending and copolymerization with PEG: FTIR investigation of molecular interactions," Biomacromolecules, vol. 4, no. 1, pp. 173-180, 2003.

[17] K. A. Howard, "Delivery of RNA interference therapeutics using polycation-based nanoparticles," Advanced Drug Delivery Reviews, vol. 61, no. 9, pp. 710-720, 2009.

[18] B. Liang, M. L. He, C.-Y. Chan et al., "The use of folate-PEGgrafted-hybranched-PEI nonviral vector for the inhibition of glioma growth in the rat," Biomaterials, vol. 30, no. 23-24, pp. 4014-4020, 2009.

[19] H. W. Sun, L. Y. Zhang, X. J. Zhu, C. Y. Kong, C. L. Zhang, and S. D. Yao, "Poly(PEGMA) magnetic nanogels: preparation via photochemical method, characterization and application as drug carrier," Science in China, Series B: Chemistry, vol. 52, no. 1, pp. 69-75, 2009.

[20] H.-W. Sun, L.-Y. Zhang, X.-J. Zhu, and X.-F. Wang, "Magnetic poly(PEGMA-MAA) nanoparticles: photochemical preparation and potential application in drug delivery," Journal of Biomaterials Science, Polymer Edition, vol. 20, no. 12, pp. 16751686, 2009.

[21] X. Liu, H. Liu, J. Xing et al., "Preparation and characterization of superparamagnetic functional polymeric microparticles," China Particuology, vol. 1, no. 2, pp. 76-79, 2003.

[22] D. Maity and D. C. Agrawal, "Synthesis of iron oxide nanoparticles under oxidizing environment and their stabilization in aqueous and non-aqueous media," Journal of Magnetism and Magnetic Materials, vol. 308, no. 1, pp. 46-55, 2007.

[23] D. M. Xu, J. Hong, H. Sun et al., "Size controlling of M-PEIS nanogels via photo-induced Fenton reaction," Acta Polymerica Sinica, vol. 4, no. 4, pp. 389-393, 2007.

[24] D. E. Sosnovik, M. Nahrendorf, and R. Weissleder, "Magnetic nanoparticles for MR imaging: agents, techniques and cardiovascular applications," Basic Research in Cardiology, vol. 103, no. 2, pp. 122-130, 2008.

[25] D. E. Sosnovik, M. Nahrendorf, N. Deliolanis et al., "Fluorescence tomography and magnetic resonance imaging of myocardial macrophage infiltration in infarcted myocardium in vivo," Circulation, vol. 115, no. 11, pp. 1384-1391, 2007.

[26] C. Xu, K. Xu, H. Gu et al., "Nitrilotriacetic acid-modified magnetic nanoparticles as a general agent to bind histidinetagged proteins," Journal of the American Chemical Society, vol. 126, no. 11, pp. 3392-3393, 2004.

[27] P. Tartaj and C. J. Serna, "Synthesis of monodisperse superparamagnetic $\mathrm{Fe} /$ silica nanospherical composites," Journal of the American Chemical Society, vol. 125, no. 51, pp. 15754-15755, 2003.

[28] H. Amiri, R. Bustamante, A. Millán et al., "Magnetic and relaxation properties of multifunctional polymer-based nanostructured bioferrofluids as MRI contrast agents," Magnetic Resonance in Medicine, vol. 66, no. 6, pp. 1715-1721, 2011.

[29] X. Yuan, Z. Luo, Q. Zhang et al., "Synthesis of highly fluorescent metal $(\mathrm{Ag}, \mathrm{Au}, \mathrm{Pt}$, and $\mathrm{Cu}$ ) nanoclusters by electrostatically induced reversible phase transfer," ACS Nano, vol. 5, no. 11, pp. 8800-8808, 2011. 

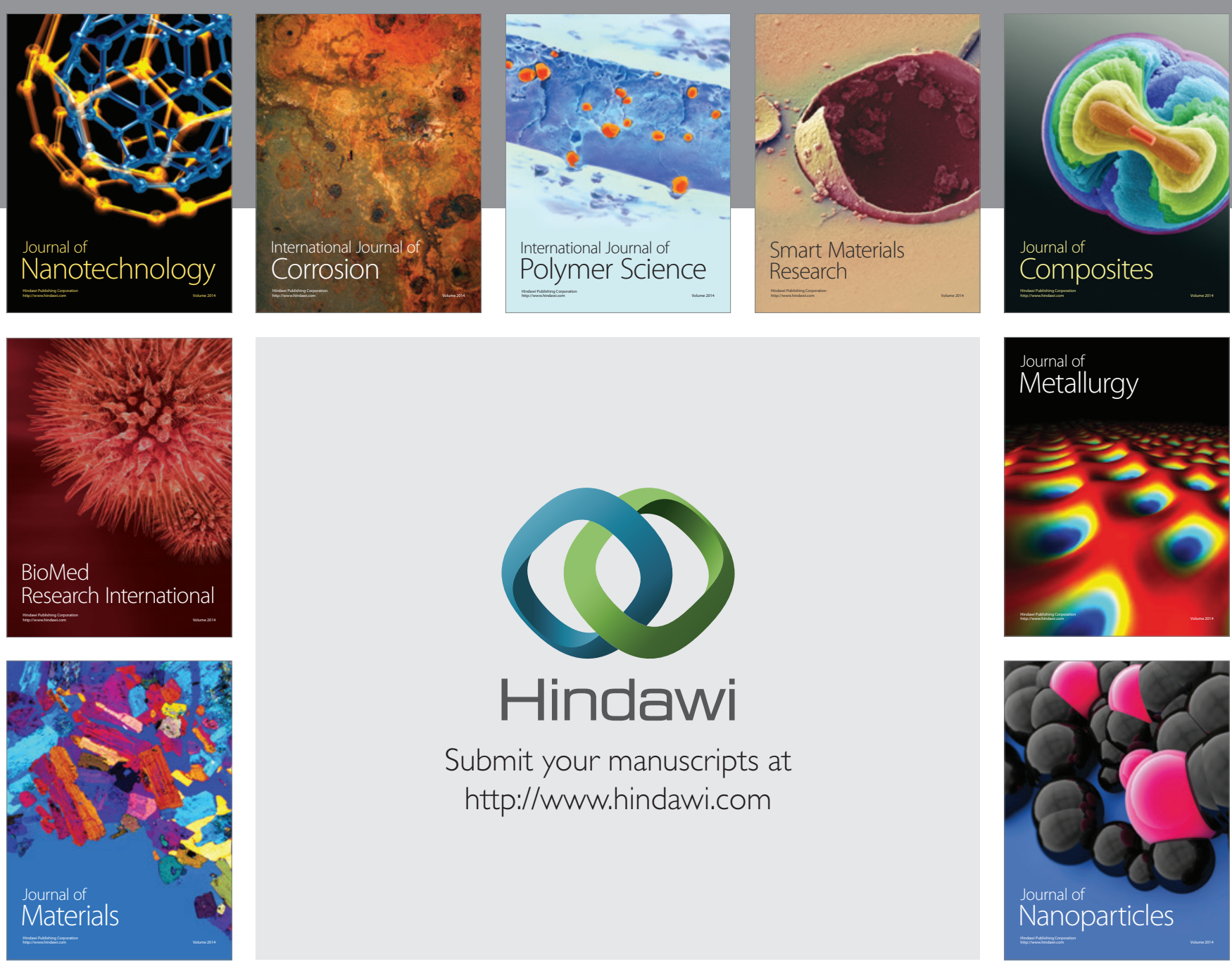

Submit your manuscripts at http://www.hindawi.com
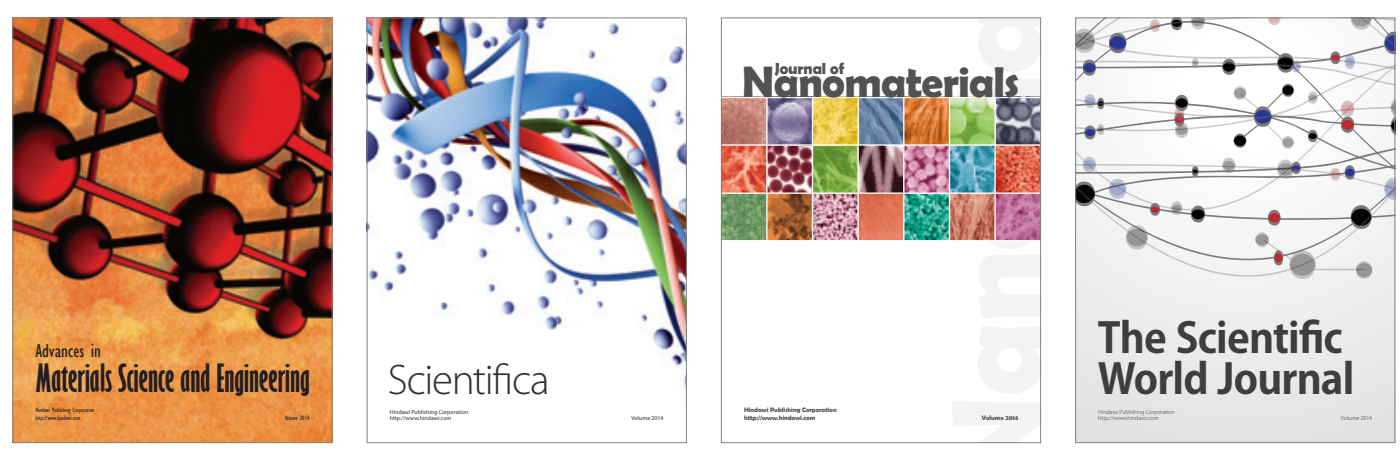

\section{The Scientific World Journal}
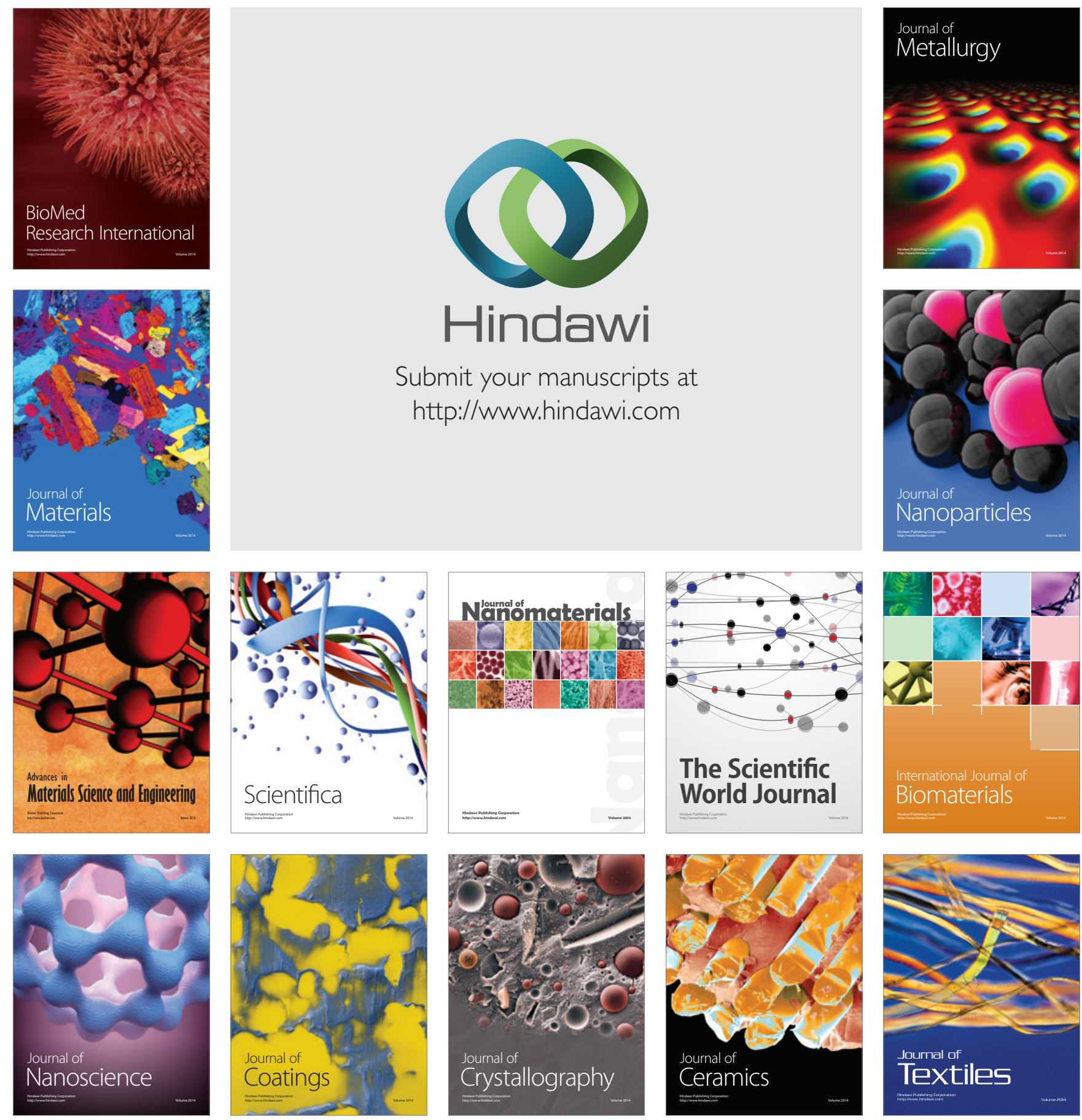